\author{
Takuzo Iwatsubo \\ Shozo Kawamura \\ Masahito Kamada \\ Department of Mechanical \\ Engineering \\ Faculty of Engineering \\ Kobe University \\ Rokkodai, Nada, Kobe 657, Japan
}

\section{Identification of Acoustic-Vibratory System by Acoustic Measurement}

A new method for reducing ill-conditioning in a class of identification problems is proposed. The key point of the method is that the identified vibration of the sound source is expressed as a superposition of vibration modes. The mathematical property of the coefficient matrix, the practical error expanding ratio, and the stochastic error expanding ratio are investigated in a numerical example. The mode-superposition method is shown to be an effective tool for acoustic-vibratory inverse analysis. (C) 1996 John Wiley \& Sons, Inc.

\section{INTRODUCTION}

The diagnosis of vibration in machines has become very important in recent years for monitoring vibrations and reducing noise. It is necessary to identify the vibrations of a machine to diagnose the vibrations further. The vibrations are measured by a vibration sensor attached directly to the machine or by a noncontact vibration sensor. When the vibration sensor is attached to a compact or a lightweight machine, however, the vibration mode changes and the identification of the vibration becomes inaccurate. In that case consideration should be given to identification of vibration by an acoustic measurement. The identification method is based on the acoustic-vibratory inverse analysis. Generally, the inverse problem becomes an ill-conditioned problem in which the accuracy of the identified values is substantially influenced by a small error present in the measured data. In acoustic-vibratory inverse analysis, the ill-conditioning problem is that the accuracy of the identified vibration decreases significantly, even if only a small error is present in the measured sound pressure data. In inverse analysis, it is impossible to completely remove the error expansion in the identified results. Therefore, it is important to reduce the error expansion from the measured data.

There are two important methods of inverse analysis. They are the method of ill-conditioning estimation and the method of ill-conditioning reduction. The condition number is generally used as an estimate of the severity of the ill-conditioning. The condition number is the ratio of the maximum singular value and the minimum non-zero singular value of the coefficient matrix. It is mathematically defined as the maximum error expanding ratio. It is not useful from the engineering viewpoint, and it does not give the practical error expanding ratio. The computation of the condition number requires extensive calculations because eigenvalue analysis has to be performed. 
There are many studies on how the error expanding ratio or the condition number should be reduced in order to reduce the ill-conditioning. Kishimoto et al. (1989) studied this point in the inverse analysis of galvanic corrosion. When the problem is particularly severely ill-conditioned, for example, when the measuring points are far from the structure, many small singular values exist in the coefficient matrix. The method of illconditioning reduction sets the very small singular values equal to zero. In this way, the minimum singular value becomes large and the condition number is reduced. Moreover, there are some studies about the threshold singular value for omission. Akaike (1974) proposed the AIC (an information criterion) value. This method is based on mathematical theory; but for acoustic-vibratory inverse analysis, a new method is considered. It uses the fact that the vibrations are expressed as a superposition of some vibration modes.

Iwatsubo et al. $(1990,1991)$ proposed an illconditioning estimation method that uses the determinant of the coefficient matrix in the boundary element method (BEM) direct method. The BEM direct method is based on an approach in which the acoustic data is measured at the same number of measuring points as the number of elements of the sound source. The vibration of the sound source is then identified by solving simultaneous linear equations. The measuring conditions to reduce the ill-conditioning are investigated numerically and experimentally. To identify the vibration accurately, however, the number of measuring points have to be increased.

In this study, a new ill-conditioning reduction method is proposed. The key to the method is that the vibration of the sound source is expressed as a superposition of the vibration modes, so that the number of identified parameters can be reduced. It also means that the number of measuring points can be reduced. In the numerical example, a simply supported rectangular plate is picked up as the sound source, and the influence of the measuring condition on the ill-conditioning is investigated. The determinant and the condition number of the coefficient matrix are used as the ill-conditioning estimation index. The result is compared with the one obtained by the BEM direct method. Next, the error expanding ratio is calculated using the measured data, in which some errors are present.
The result is compared with the one obtained by the BEM direct method. Finally, the new error expanding ratio is described.

\section{FORMULATION OF ACOUSTIC-VIBRATORY INVERSE PROBLEM}

\section{Wave Equation}

To explain the acoustic-vibratory inverse problem, a sound source model shown in Fig. 1 is considered. The governing equation for the acoustic field is the wave equation

$$
\frac{\partial^{2} P(\mathbf{x}, t)}{\partial^{2} t}=c^{\prime \prime} \nabla^{2} P(\mathbf{x}, t),
$$

where $P(\mathbf{x}, t)$ is the sound pressure, $c$ is the sound velocity, and $\mathbf{x}$ is the position vector of the measuring point. If it is assumed that the sound pressure behaves harmonically with the angular frequency $\omega$, the governing equation becomes the Helmholtz equation:

$$
\nabla^{2} p(\mathbf{x})+k^{2} p(\mathbf{x})=0,
$$

where $p(\mathbf{x})$ is the magnitude of sound pressure and $k$ is the wave number defined as $\omega / c$. The boundary condition on the boundary $\Gamma$, that is the surface of the sound source, is as follows:

$$
\frac{\partial \bar{p}(\mathbf{y})}{\partial \mathbf{n}(\mathbf{y})}=-j \omega \rho \bar{v}(\mathbf{y}) \equiv \bar{q}(\mathbf{y}),
$$

where $\mathbf{n}$ is the vector normal to the surface, $\rho$ is the density of air, $\bar{v}$ is the velocity on the surface, $\mathbf{y}$ is the position vector on the surface, and the overbar is the value of the variable on the surface.

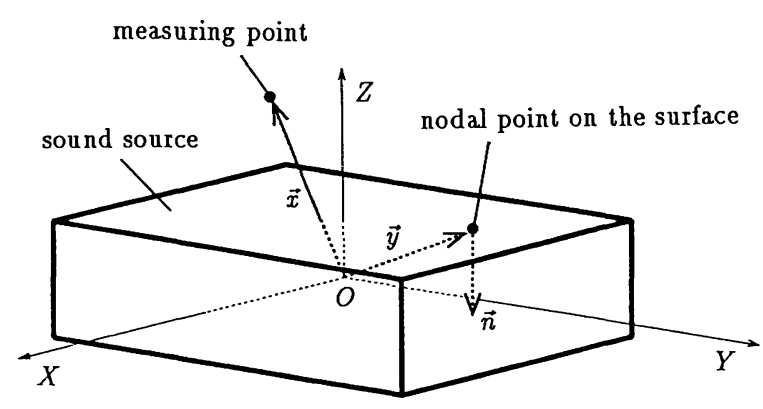

FIGURE 1 Sound source. 
If Eq. (2) is transformed by Green's formula, the boundary integral equations are obtained as follows:

$$
\begin{aligned}
p(\mathbf{x})= & \int_{\Gamma}\left(p^{*}\left(\mathbf{x}, \mathbf{y}^{\prime}\right) \bar{q}\left(\mathbf{y}^{\prime}\right)\right. \\
& \left.-q^{*}\left(\mathbf{x}, \mathbf{y}^{\prime}\right) \bar{p}\left(\mathbf{y}^{\prime}\right)\right) d \Gamma\left(\mathbf{y}^{\prime}\right), \\
\frac{1}{2} \bar{p}(\mathbf{y})= & \int_{\Gamma}\left(p^{*}\left(\mathbf{y}, \mathbf{y}^{\prime}\right) \bar{q}\left(\mathbf{y}^{\prime}\right)\right. \\
& \left.-q^{*}\left(\mathbf{y}, \mathbf{y}^{\prime}\right) \bar{p}\left(\mathbf{y}^{\prime}\right)\right) d \Gamma\left(\mathbf{y}^{\prime}\right),
\end{aligned}
$$

where $p^{*}(\mathbf{x}, \mathbf{y})$ is the basic solution of Eq. (2)

$$
p^{*}(\mathbf{x}, \mathbf{y})=\frac{1}{4 \pi r} \exp (-i k r), \quad q^{*}(\mathbf{x}, \mathbf{y}) \equiv \frac{\partial p^{*}(\mathbf{x}, \mathbf{y})}{\partial \mathbf{n}(\mathbf{y})},
$$

where $r$ is the distance between $\mathbf{x}$ and $\mathbf{y}$.

To analyze Eq. (4) numerically, the surface of the sound source is divided into $n$ elements, and it is assumed that $\bar{p}(\mathbf{y})$ and $\bar{q}(\mathbf{y})$ are constant on each element. Then the boundary integral equation, Eq. (4), can be distributed as

$$
\begin{aligned}
\{p\} & =[G]\{\bar{v}\}-[H]\{\bar{p}\}, \\
\frac{1}{2}\{p\} & =[\bar{G}]\{\bar{v}\}-[\bar{H}]\{\bar{p}\},
\end{aligned}
$$

where $\{p\}$ is the $m$-dimensional vector expressing the sound pressure at $m$ measuring points, and $\{\bar{p}\}$ and $\{\bar{v}\}$ are the sound pressure and velocity vector at $n$ nodal points on the surface. By eliminating $\{\bar{p}\}$, the following simultaneous linear equations are obtained.

$$
\begin{aligned}
\{p\} & =\left\{[G]-[H]\left(\frac{1}{2}[I]+[\bar{H}]\right)^{-1}\right. \\
{[\bar{G}]\}\{\bar{v}\} } & \equiv[S]\{\bar{v}\}
\end{aligned}
$$

where $[S]$ is the constant matrix determined by the number of elements, the measuring condition, and so on. These procedures have been described in Schenck (1968).

By using Eq. (7), the sound pressure in space can be calculated by measuring the velocity on the surface. For the inverse problem in this study, however, the vibration $\{\bar{v}\}$ on the sound source has to be identified by measuring the sound pressure $\{p\}$ in space. Therefore, in the previous method the number of the measuring points of the sound pressure is the same as the number of the elements of the sound source ( $m=$ n). Then Eq. (7) becomes

$$
\{\bar{v}\}=[S]^{-1}\{p\} .
$$

This method was called the BEM direct method by Iwatsubo et al. $(1990,1991)$. The ill-conditioning problem is that the error in the measured sound pressure data $\{p\}$ is expanded and the accuracy of the identified vibration data $\{\bar{v}\}$ deteriorates.

\section{Formulation by Superposition of Vibration Modes}

The mode-superposition method consists of reducing the number of identified parameters, and the ill-conditioning is reduced by using the principle of superposition.

The eigenvalue analysis of the sound source is carried out first. Distributing the sound source by the finite element method (FEM), the mass matrix $[M]$ and the stiffness matrix $[K]$ are obtained. The eigenvalue analysis is performed using the matrices as follows:

$$
\left([K]-\omega_{i}^{2}[M]\right)\left\{\phi_{i}\right\}=\{0\},
$$

where $\omega_{i}$ and $\left\{\phi_{i}\right\}$ are the eigenfrequency and mode vector for the $i$ th vibration mode, respectively.

Here the steady-state vibration vector $\{\bar{X}(\mathbf{y}$, $t)\}$ with the angular frequency $\omega$ is expressed as

$$
\{\bar{X}(\mathbf{y}, t)\}=\{\bar{X}(\mathbf{y})\} \exp (j \omega t) .
$$

The vector $\{\bar{X}(\mathbf{y})\}$ can be expressed by the superposition of mode vectors $\{\phi\}$. Next the velocity vector $\{\bar{V}(\mathbf{y}, t)\}$ is expressed as

$$
\{\bar{V}(\mathbf{y}, t)\}=j \omega\{\bar{X}(\mathbf{y})\} \exp (j \omega t) \equiv\{\bar{v}(\mathbf{y})\} \exp (j \omega t),
$$

and the vector $\{\bar{v}(\mathbf{y})\}$ is expressed by the superposition of mode vectors as

$$
\{\bar{v}(\mathbf{y})\}=\sum_{k=1}^{\infty} A_{k}\left\{\phi_{k}(\mathbf{y})\right\}=[\Phi]\{A\},
$$

where $\{\bar{v}(\mathbf{y})\}$ is the velocity vector at the position $\mathbf{y},[\Phi]$ is the modal matrix that consists of the mode vectors of the structure, and $\{A\}$ is the 
vector that consists of the influence coefficients for every mode vector. They are expressed as follows:

$$
\begin{aligned}
\{\bar{v}\} & =\left\{\bar{v}\left(\mathbf{y}_{1}\right), \bar{v}\left(\mathbf{y}_{2}\right), \ldots, \bar{v}\left(\mathbf{y}_{n}\right)\right\}^{T} \\
\{A\} & =\left\{A_{1}, A_{2}, \ldots, A_{x}\right\}^{T} \\
{[\Phi] } & =\left[\begin{array}{cccc}
\phi_{1}\left(\mathbf{y}_{1}\right) & \phi_{2}\left(\mathbf{y}_{1}\right) & \cdots & \phi_{x}\left(\mathbf{y}_{1}\right) \\
\vdots & \vdots & \ddots & \vdots \\
\phi_{1}\left(\mathbf{y}_{n}\right) & \phi_{2}\left(\mathbf{y}_{n}\right) & \cdots & \phi_{x}\left(\mathbf{y}_{n}\right)
\end{array}\right] .
\end{aligned}
$$

When Eq. (12) is substituted into Eq. (7), the following equation is obtained:

$$
\{p\}=[S][\Phi]\{A\} \equiv\left[S_{M}\right]\{A\} .
$$

In this way, the acoustic-vibratory inverse problem is formulated as a problem in which the influence coefficient $\{A\}$ is identified by measuring the sound pressure $\{p\}$. Generally, it is considered that the vibration of a structure is dominated by a small number of the vibration modes. Accordingly, the influence coefficients can be reduced by using the vibration modes included in the dominant frequency band. It is considered that the ill-conditioning can be reduced because the coefficient matrix $\left[S_{M}\right]$ consists of the dominant vibration mode vectors. It means also that the number of the measuring points can be reduced. For example, if the vibration of the sound source is expressed by $q$ vibration modes, then Eq. (14) are $n$ equations including $q$ unknown parameters $A_{k}(k=1, \ldots, q)$. When Eq. (14) is solved by the method of least squares, the following equation is obtained:

$$
\{A\}=\left(\left[S_{M}\right]^{T}\left[S_{M}\right]\right)^{-1}\left[S_{M}\right]^{T}\{p\} .
$$

Moreover, the velocity of the sound source is calculated by using Eq. (12).

$$
\{\bar{v}(\mathbf{y})\}=[\Phi]\left(\left[S_{M}\right]^{T}\left[S_{M}\right]\right)^{-1}\left[S_{M}\right]^{T}\{p\} \equiv[Q]\{p\} .
$$

\section{ILL-CONDITIONING REDUCTION BY MODE SUPERPOSITION}

When the inverse problem is solved by using the measured data, the error expanding ratio is influ- enced by the mathematical property of the coefficient matrix.

In this chapter, the mathematical property of the coefficient matrix and the error expanding ratio are compared between the mode-superposition method and the BEM direct method.

\section{Mathematical Property of Coefficient Matrix}

The mathematical property of the coefficient matrix is investigated by using the "determinant" and "condition number" as the ill-conditioning index.

The determinant is used as the index because the ill-conditioning can be considered mathematically as a singularity condition. It is defined in this article as $\operatorname{det}\left(\left[S_{M}\right]^{T}\left[S_{M}\right]\right)$. If the determinant is large, the ill-conditioning can be reduced.

The condition number $\mu$ is mathematically defined as the ratio between the maximum singular value $\kappa_{\max }$ and the minimum, but nonzero, singular value $\kappa_{\min }$ of the coefficient matrix expressed as follows:

$$
\mu=\frac{\kappa_{\max }}{\kappa_{\min }} .
$$

The condition number is the maximum error expanding ratio expressed as:

$$
\frac{\|\{\delta \tilde{v}\}\|}{\| \tilde{v}\} \|} \leq \mu \cdot \frac{\|\{\delta p\}\|}{\|\{p\}\|},
$$

where the value within the vertical bars is the Euclid norm of the value. However, the practical error expanding ratio seldom agrees with the condition number.

The property of the coefficient matrix is investigated using the above indicators. The following four measuring conditions are considered: the frequency of the sound source, the distance between the measuring point and the sound source, the numer of the measuring points, and the number of the adoptive vibration modes. The distance between the measuring point and the sound source is expressed by the nondimensional distance $r_{z}$. This value is the vertical distance from the surface of the sound source to the measuring point divided by the characteristic length $r_{a}$, which is the square root of the surface area of the sound source. The vibration modes around the frequency $\omega$ of the vibration are used as the adoptive vibration modes. 


\section{Error Expanding Ratio Using Measured Data}

Ill-conditioning can be estimated by using the de terminant or the condition number given in the last section. In practice, however, the error expanding ratio is also important because the error expanding ratio is closely related to the accuracy of the identified value. Therefore the error expanding ratio is investigated by using the Monte Carlo simulation. The relation between the illconditioning index and the error expanding ratio is also investigated.

The procedures are as follows. First, the case in which the sound source is harmonically excited with the angular frequency $\omega$ is considered. Then the exact velocity $v_{\mathrm{ex}}$ on the sound source is calculated by numerically integrating the equation of motion. Next, the positions and the number of measuring points are determined. The exact sound pressures $p_{\mathrm{ex}}$ can be calculated by using Eq. (7). The measured sound pressure data with error is obtained by adding random numbers to the exact sound pressure data.

Generating $M$ numbers of $\tilde{p}$ at each measuring point, $M$ sets of the sound pressure data $\{\tilde{p}\}_{l}$ $(l=1, \ldots ., M)$ are made. In the case of the mode-superposition method, $M$ sets of the influence coefficient $\{A\}_{l}(l=1, \ldots, M)$ are calculated by Eq. (15), and $M$ sets of the identified results $\{\tilde{v}\}_{l}(l=1, \ldots, M)$ are calculated by Eq. (12). In the case of the BEM direct method, $M$ sets of the identified results $\{\tilde{v}\}_{l}(l=1, \ldots$, $M)$ are calculated by Eq. (8). At each nodal point, the average $|\tilde{v}|_{\text {av }}$ and the standard deviation $\sigma_{|\tilde{v}|}$ of the amplitude of the velocity $\tilde{v}$ are obtained, and the average $\angle \tilde{v}_{\mathrm{av}}$ and the standard deviation $\sigma_{\angle \bar{v}}$ of the phase of the velocity are obtained. The coefficients of variation $\sigma_{|\tilde{v}|} /|\tilde{v}|_{\mathrm{av}}$ and $\sigma_{\angle \tilde{v}} / \angle \tilde{v}_{\mathrm{av}}$ are calculated and compared with the error in the measured data.

\section{Stochastic Error Expanding Ratio}

The error expanding ratio based on a stochastic method of calculation is described in this section.

The velocity at the $i$ th nodal point $v_{i}$ is expressed by Eq. (16) as:

$$
v_{i}=\sum_{j=1}^{n} q_{i j} \cdot p_{j},
$$

where $q_{i j}$ and $p_{j}$ are the elements of the matrix [Q] and vector $\{p\}$, respectively. The average of $v_{i}$ is approximated as follows;

$$
\left(v_{i}\right)_{\mathrm{av}}=\sum_{j=1}^{n} q_{i j} \cdot\left(p_{j}\right)_{\mathrm{av}},
$$

and the standard deviation is approximated as:

$$
\left(\sigma_{v_{i}}\right)^{2}=\sum_{j=1}^{n}\left(q_{i j}\right)^{2} \cdot\left(\sigma_{p_{j}}\right)^{2} .
$$

If the coefficient of variation of the pressure data is assumed to be constant at all the measuring points, the coefficient of variation of the velocity is obtained using Eqs. (19) and (20) as:

$$
\begin{aligned}
\left(\frac{\sigma_{v_{i}}}{\left(v_{i}\right)_{\mathrm{av}}}\right)^{2} & =\frac{\sum_{j=1}^{n}\left(q_{i j}\right)^{2} \cdot\left(\sigma_{p_{j}}\right)^{2}}{\left(\sum_{j=1}^{n}\left(q_{i j} \cdot p_{j}\right)^{2}\right.} \\
& =\frac{\sum_{j=1}^{n}\left(q_{i j} \cdot p_{j}\right)^{2}}{\left(\sum_{j=1}^{n} q_{i j} \cdot p_{j}\right)^{2}} \cdot\left(\frac{\sigma_{p}}{p}\right)^{2} \equiv R_{i}^{2} \cdot\left(\frac{\sigma_{p}}{p}\right)^{2} .
\end{aligned}
$$

In this way, the coefficient of variation of the velocity can be expressed by using the coefficient of variation of the sound pressure data. The coefficient $R_{i}$ is considered as the stochastic error expanding ratio. The magnitude of $R_{i}$ is compared with the error expanding ratio by using the Monte Carlo simulation.

\section{NUMERICAL EXAMPLES}

As a sound model, the simply supported rectangular plate $(16 \mathrm{~cm} \times 12 \mathrm{~cm} \times 0.5 \mathrm{~mm})$ shown in Fig. 2 is picked up. The dimensions of the plate are shown in Table 1 . The surface of the model is divided into 32 triangle elements. The natural frequencies and the vibration modes of the model

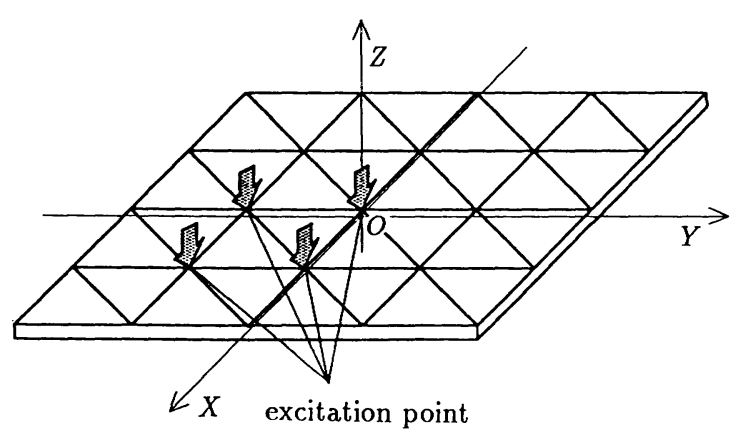

FIGURE 2 Rectangular plate. 
Table 1. Dimensions of Sound Source

\begin{tabular}{|l|ll|}
\hline Length & $1.6 \times 10^{-1} \quad[\mathrm{~m}]$ \\
\hline Width & $1.2 \times 10^{-1}$ & {$[\mathrm{~m}]$} \\
\hline Thickness & $5.0 \times 10^{-4}$ & {$[\mathrm{~m}]$} \\
\hline Modulus of elasticity & $2.05 \times 10^{+11}$ & {$[\mathrm{~Pa}]$} \\
\hline Poisson's ratio & 0.3 & \\
\hline Mass density & $7.86 \times 10^{+3}$ & {$\left[\mathrm{~kg} / \mathrm{m}^{3}\right]$} \\
\hline
\end{tabular}

are calculated. Table 2 shows some of the natural frequencies.

\section{Mathematical Property of Coefficient Matrix}

The property of the coefficient matrix is investigated using the determinant and the condition number. The four measuring conditions, shown in the last section, are considered. Here the frequency of the sound source is set as 100,300 , and $500 \mathrm{~Hz}$. The adoptive vibration modes are the ones whose natural frequencies are near the frequency of the sound source. Table 3 shows the adoptive vibration modes for the frequency of the sound source. The sound pressures are measured at five or nine points shown in Fig. 3. The determinant and the condition number are calculated and compared.

Figure 4(a)-(d) shows the change of the determinant, $\operatorname{det}\left(\left[S_{M}\right]^{T}\left[S_{M}\right]\right)$, when the measuring distance, the number of the measuring points, and the number of the adoptive vibration modes are changed for three frequencies.

Figure 4 shows the following:

1. When the frequency of the sound source is higher or when the measuring points are closer to the sound source, the ill-conditioning can be reduced.

Table 2. Natural Frequencies

\begin{tabular}{|c|c|}
\hline & Natural Frequency $[\mathrm{Hz}]$ \\
\hline$f_{1}$ & 133.3 \\
\hline$f_{2}$ & 275.7 \\
\hline$f_{3}$ & 385.4 \\
\hline$f_{4}$ & 510.6 \\
\hline$f_{5}$ & 520.8 \\
\hline$f_{6}$ & 731.2 \\
\hline
\end{tabular}

Table 3. Adoptive Vibration Modes

\begin{tabular}{|c|c|}
\hline Frequency $\omega[\mathrm{Hz}]$ & Vibration Modes \\
\hline 100 & $\left\{\phi_{1}\right\},\left\{\phi_{2}\right\},\left\{\phi_{3}\right\},\left\{\phi_{4}\right\}, \cdots$ \\
\hline 300 & $\left\{\phi_{2}\right\},\left\{\phi_{3}\right\},\left\{\phi_{1}\right\},\left\{\phi_{4}\right\}, \cdots$ \\
\hline 500 & $\left\{\phi_{4}\right\},\left\{\phi_{5}\right\},\left\{\phi_{3}\right\},\left\{\phi_{2}\right\}, \cdots$ \\
\hline
\end{tabular}

2. Comparing Fig. 4(a) and (b), when the number of the measuring points is larger, with the same number of adoptive vibration modes, the ill-conditioning can be reduced.

3. Comparing Fig. 4(b), (c), and (d), even if the number of the adoptive vibration modes is larger, the ill-conditioning cannot necessarily be reduced. It is basically possible that the number of the adoptive vibration modes be as high as the number of the measuring points. Sometimes, however, an increase in the number of the adoptive vibration modes causes ill-conditioning. Therefore, in practical applications, the selection of the adoptive vibration modes is important.

Figure 4(e) shows the variation of the determinant $[S]$ in the BEM direct method. From this
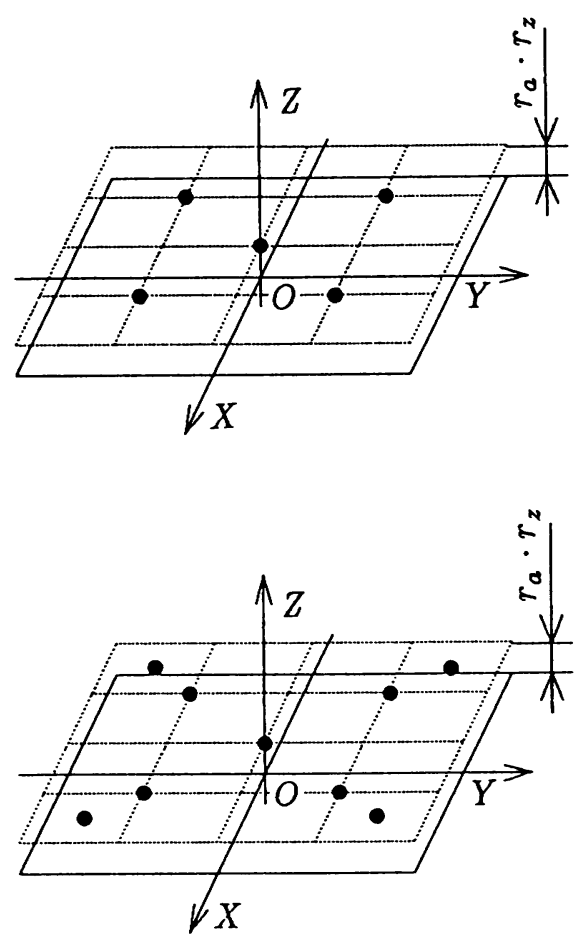

FIGURE 3 Arrangement of measuring points: (a) 5 measuring points and (b) 9 measuring points. 

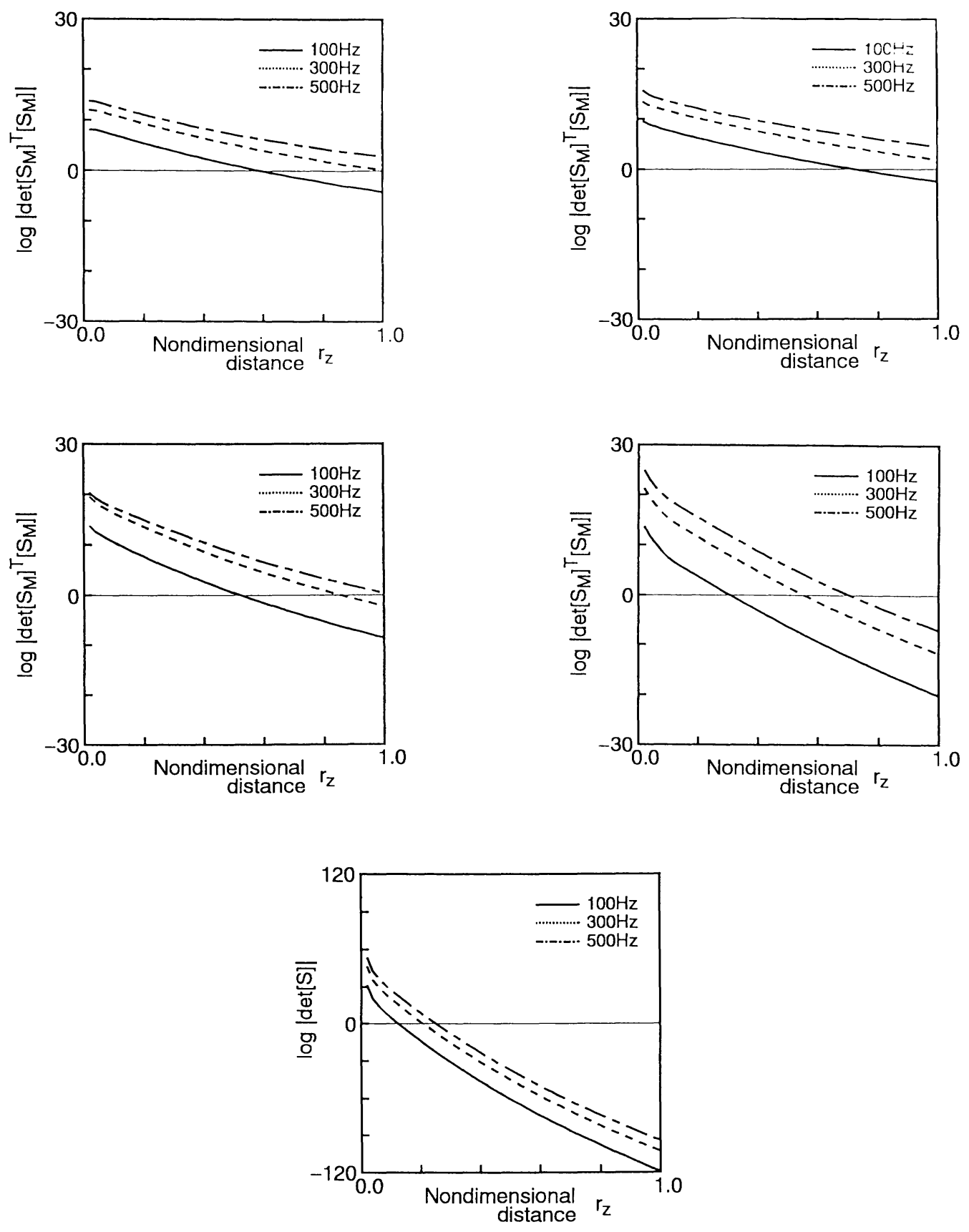

FIGURE 4 Variation of $\operatorname{det}\left(\left[S_{M}\right]^{T}\left[S_{M}\right]\right)$ : (a) 4 adoptive vibration modes, 5 measuring points; (b) 4 adoptive vibration modes, 9 measuring points; (c) 6 adoptive vibration modes, 9 measuring points; (d) 8 adoptive vibration modes, 9 measuring points; and (e) BEM direct method, 32 measuring points.

figure, the variation of the measuring distance influences the ill-conditioning considerably in the BEM direct method.

The variation of the condition number $\mu$ is shown in Fig. 5. From the figure, similar results are obtained as with the determinant. It has been found, however, that the condition number is more influenced by the adoptive vibration modes than by the frequency of the sound source.

It is found that the ill-conditioning can be reduced by using the necessary minimum number of adoptive vibration modes and many measuring 

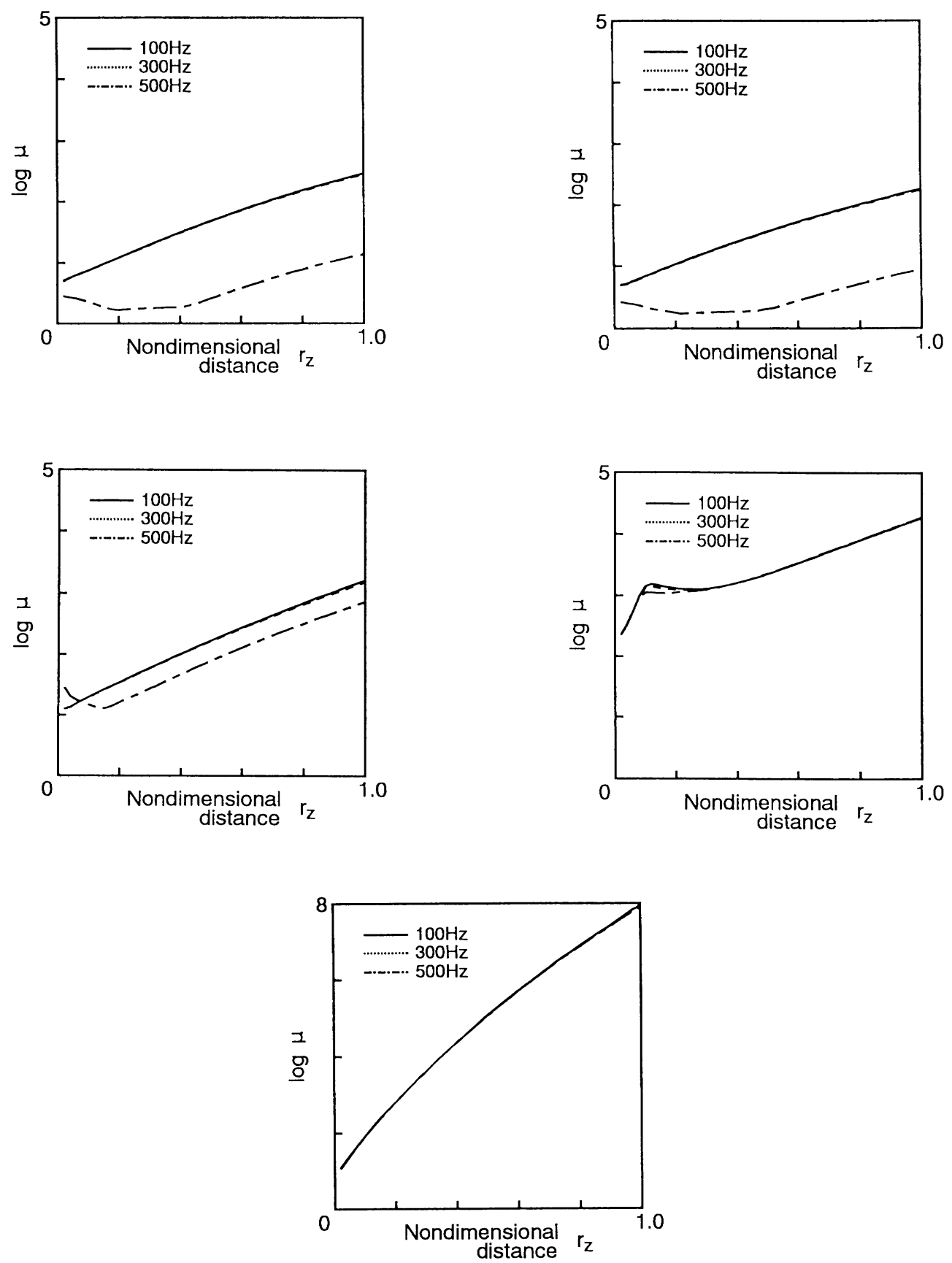

FIGURE 5 Variation of condition number $\mu$ : (a) 4 adoptive vibration modes, 5 measuring points; (b) 4 adoptive vibration modes, 9 measuring points; (c) 6 adoptive vibration modes, 9 measuring points; (d) 8 adoptive vibration modes, 9 measuring points; and (e) BEM direct method, 32 measuring points.

points. In practice it is necessary that the best measuring condition is found considering the model shape, the required frequency band, and so on. In this numerical example, due to good accuracy, the ill-conditioning can be reduced when six adoptive vibration modes and nine measuring points are used. It can be also reduced when four adoptive vibration modes and five measuring points are used for $\omega=100$ or $300 \mathrm{~Hz}$.

\section{Error Expanding Ratio Using Measured Data}

The error expanding ratio is investigated when the measured sound pressure data has some er- 
ror. The case in which the rectangular plate is excited harmonically at the excitation points shown in Fig. 2 is considered. The magnitude and frequency of the external force are set as 1.0 $\mathrm{N}$ and $300 \mathrm{~Hz}$, respectively. In the mode-superposition method, the number of the adoptive vibration modes is four and the number of the measuring points is five. In the BEM direct method, the number of measuring points is 32 .
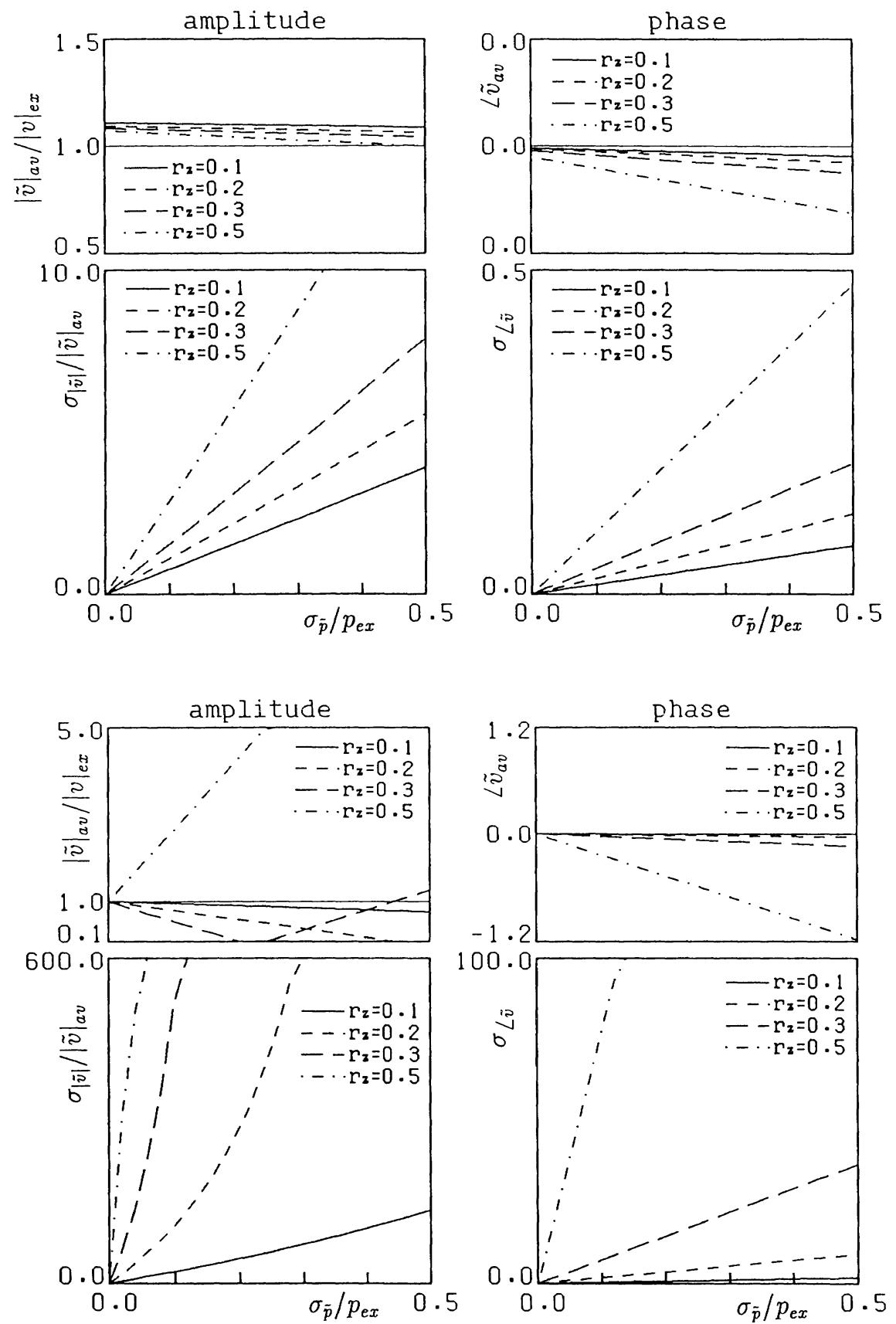

FIGURE 6 Error expanding ratio by Monte Carlo simulation: (a) mode-superposition method and (b) BEM direct method.
The coefficient of variation $\sigma_{\tilde{p}} / p_{\mathrm{ex}} \equiv \sigma_{|\bar{p}|} /|p|=$ $\sigma_{\angle \dot{p}} / \angle p$ of the normal random number, which is added to the exact value, is changed from 0.0 to 0.5. The sample number $M$ of the Monte Carlo simulation is 1000 , and the velocity at the center of the sound source model is picked up as a representation. The result is shown in Fig. 6. In the mode-superposition method, the average of the amplitude and the phase almost agrees with the

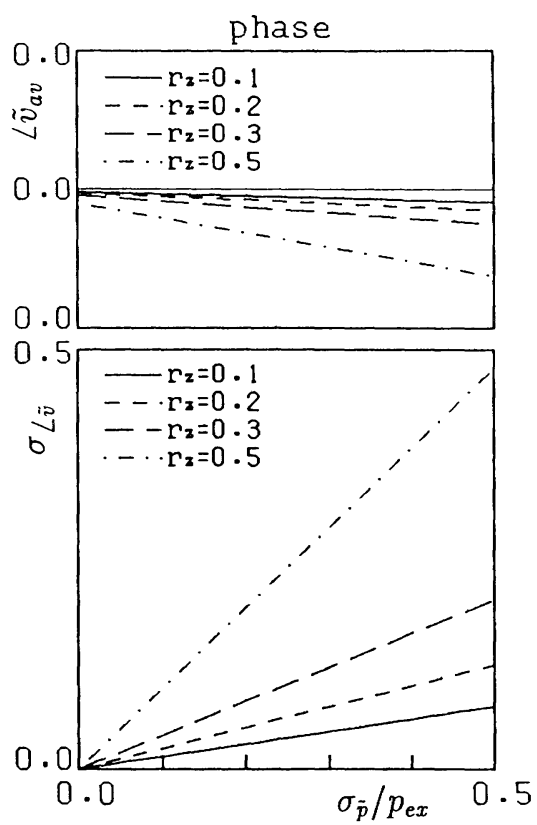


exact value. In the BEM direct method, the average amplitude is close to the exact value when $r_{z}=0.1$; but the accuracy of the identified values is rather poor when $r_{z}>0.1$, and the accuracy when $r_{z}=0.5$ is very poor. Regarding the coefficient of the variation of the amplitude and the standard deviation of the phase, in both methods the value is large when the nondimensional distance is large. The variation in the BEM direct method is especially larger than one in the modesuperposition method. This agrees with the results in the last section that the determinant and the condition number are larger in case of the BEM direct method than in the mode-superposition method. The identified values in the BEM direct method are likely to be influenced by the measuring distance. This also agrees with the results in the last section.

The accuracy of the sound pressure data when some errors are present in the measuring distance data has been investigated in the same way. It is shown that the variation of the sound pressure data is smaller than that of the measuring distance data.

As a result of this simulation, when some errors are present in the sound pressure data, the closer the measuring distance is to the sound source, the smaller the change of the variation of the velocity is. The mode-superposition method is more suitable for solving the inverse problem by using the measured data that includes some errors than the BEM direct method.

\section{Stochastic Error Expanding Ratio}

The coefficient $R$ in Eq. (22) at the center of the plate is investigated by using the same sound model and the same measuring condition as the above. It is assumed, however, that the phase of the velocity is 45 because if the phase is zero, the coefficient of variation cannot be calculated. The coefficient of variation of the pressure data $\sigma_{p} / p$ is constant and equal to 0.1 . The result is shown in Fig. 7. The broken line expresses the expanding ratio calculated from the result of the Monte Carlo simulation. It is considered to be a practical expanding ratio. In Fig. 7, the coefficient $R$ calculated by using Eq. (22) is close to the practical expanding ratio. This coefficient can be used as an indicator of accuracy of the identified values. If the measured sound pressure data is sufficiently reliable, the accuracy of the identified values, when some errors are present in the
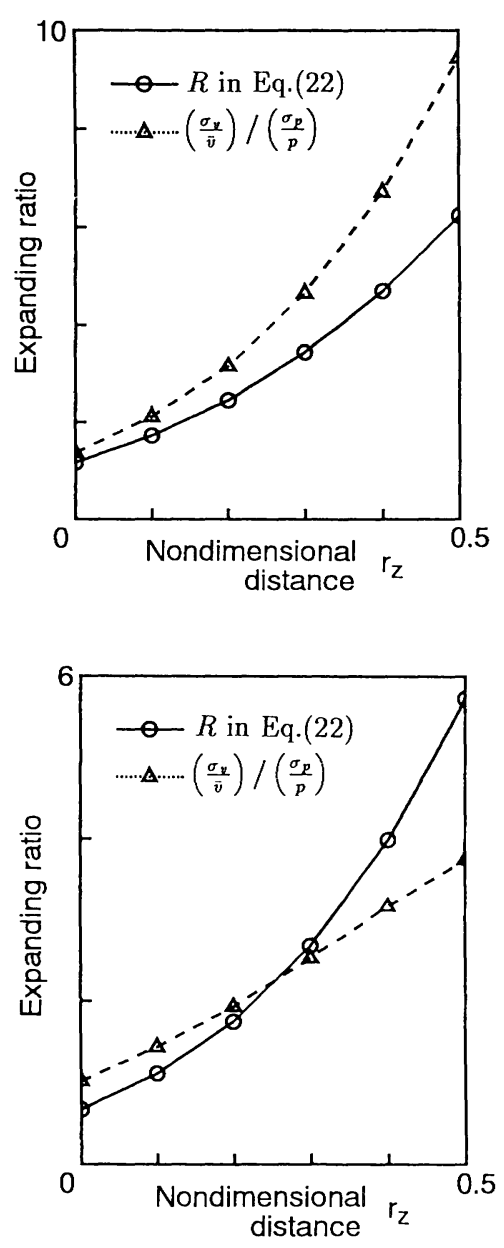

FIGURE 7 Stochastic error expanding ration: (a) real part and (b) imaginary part.

measured sound pressure data, can be predicted by calculating the coefficient $R$ in Eq. (22).

\section{CONCLUSIONS}

A new ill-conditioning reduction method has been proposed. The key point of the method is that the identified vibration of the sound source is expressed as a superposition of the vibration modes. In the numerical example, a simply supported rectangular plate is chosen as the sound source. The mathematical property of the coefficient matrix was investigated first by using the determinant and the condition number. The following results were obtained:

1. When the frequency of the sound source is higher, or when the measuring points are 
closer to the sound source, the ill-conditioning can be reduced.

2. When the number of the measuring points is larger with the same number of the adoptive vibration modes, the ill-conditioning can be reduced.

3. Even if the number of the adoptive vibration modes is larger, the ill-conditioning cannot necessarily be reduced.

4. In the BEM direct method, changes in the measuring distance influence the ill-conditioning considerably.

The error expanding ratio was investigated when the sound pressure data include some error. It was found that the closer the measuring distance is to the sound source, the smaller the change of the variation of the velocity is. The mode-superposition method is more suitable for solving the inverse problem when using measured data with some errors than the BEM direct method. Finally the stochastic error expanding ratio was investigated. The ratio was found to be close to the practical expanding ratio, and the ratio can be used as an index of the accuracy of the identified values. Therefore, the mode-superposition method is shown to be an effective method in acoustic-vibratory inverse analysis.

\section{REFERENCES}

Akaike, H., 1974, "A New Look at the Statistical Model Identification," IEEE Transactions on Automatic Control, Vol. AC-19, pp. 716-723.

Iwatsubo, T., Kawamura, S., and Kugo, S., 1990, "An Analysis of Acoustic Inverse Problem by Using Boundary Element Method," Topics of Engineering Advances in Boundary Element Methods in Japan and USA, Vol. 7, pp. 351-361.

Iwatsubo, T., Kawamura, S., and Kugo, S., 1991, "Inverse Analysis of Vibratory-Acoustic System by Using Boundary Element Method," Proceedings of the 1991 Asia-Pacific Vibration Conference, Vol. 2, pp. 11.8-11.13.

Kishimoto, K., Miyasaka, H., and Aoki, S., 1989, "Boundary Element Analysis of an Inverse Problem in Galvanic Corrosion," JSME International Journal, Vol. 32, pp. 256-262.

Schenck, H. A., 1968, "Improved Integral Formulation for Acoustic Radiation Problems," The Journal of the Acoustical Society of America, Vol. 44, pp. 41-58. 

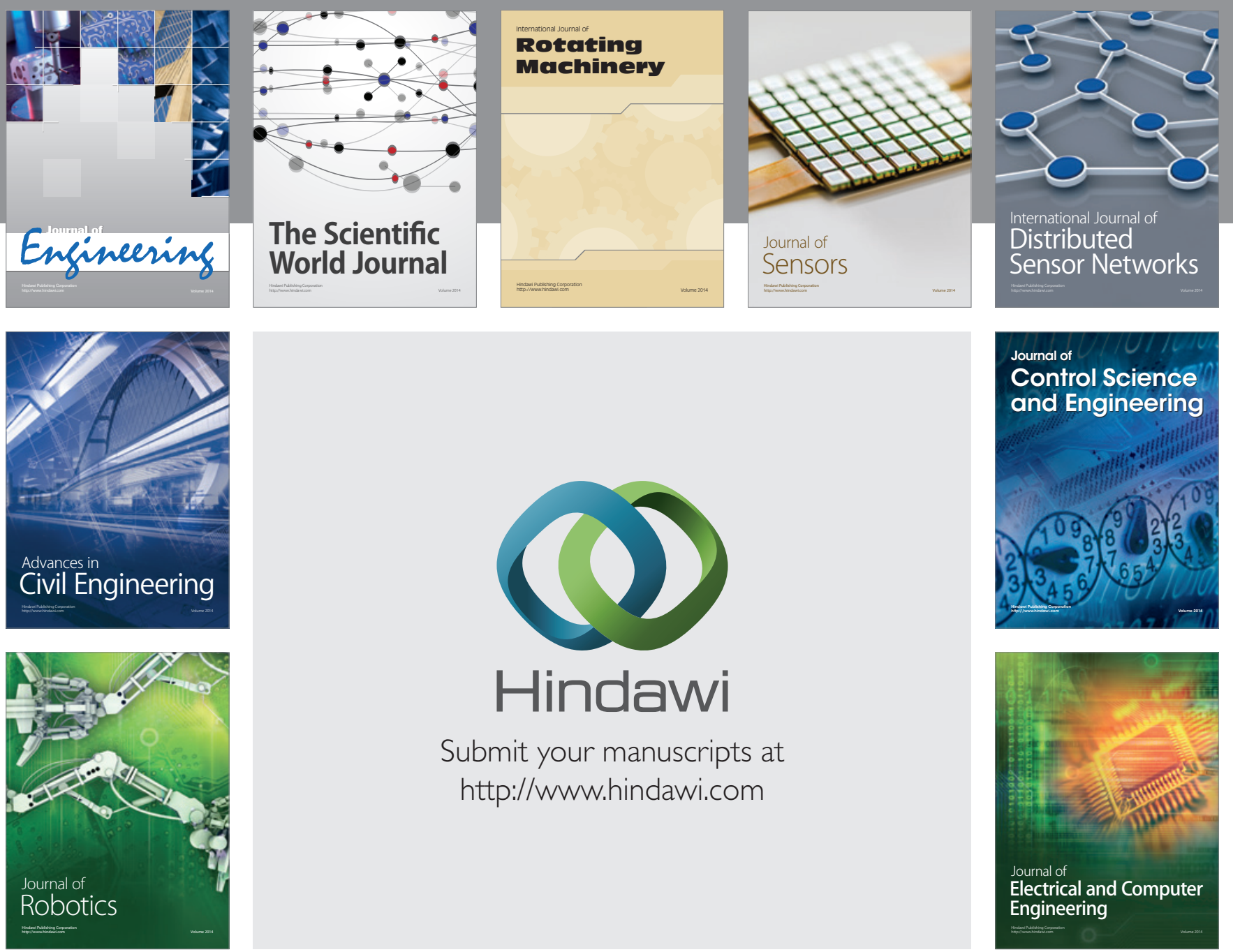

Submit your manuscripts at

http://www.hindawi.com
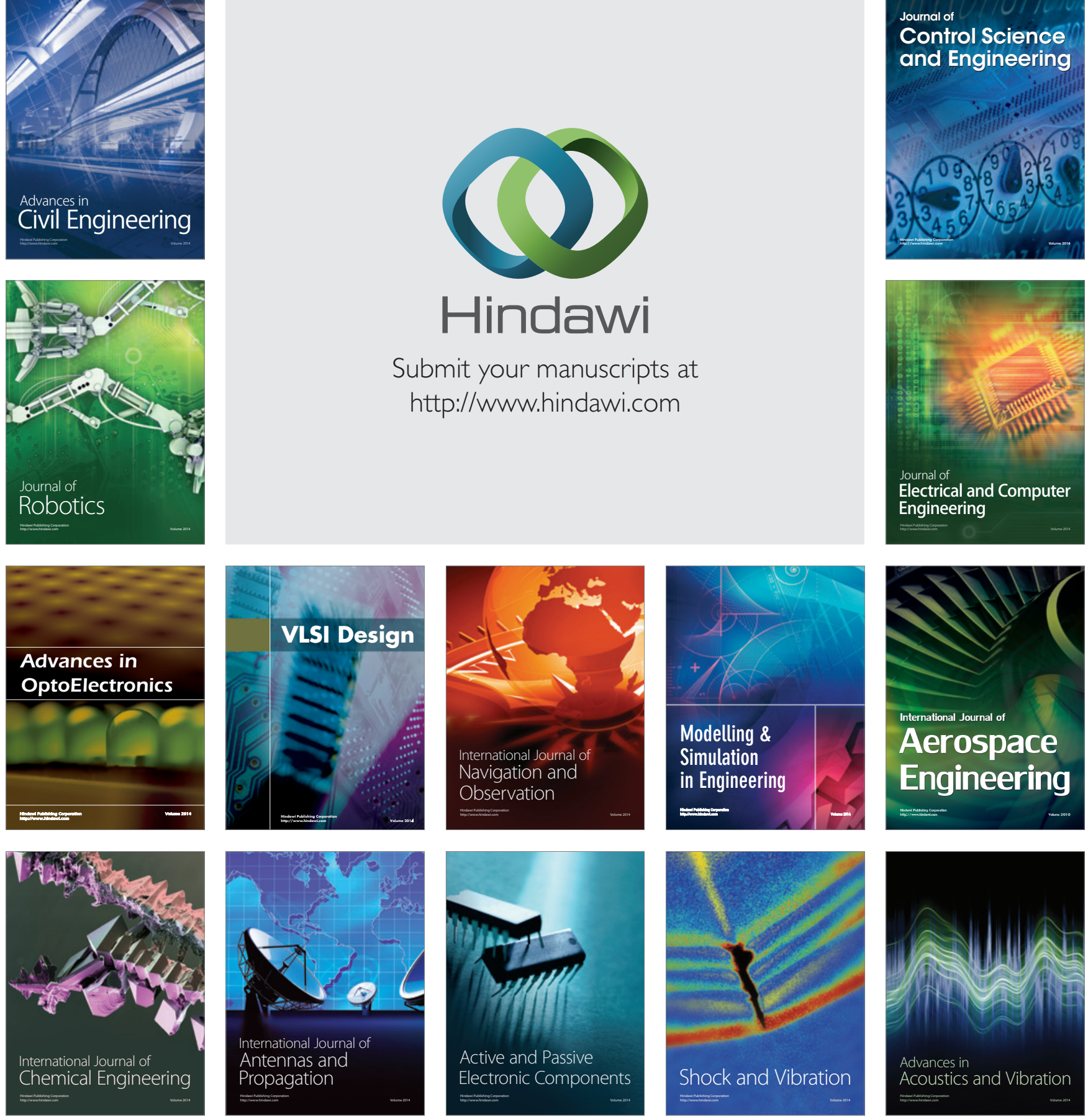